\title{
Development of Embedded Web Server Based on DAC System Using ARM for Industrial Applications. \\ Dr.M.Kamaraju', D.Gopinath ${ }^{2}$ \\ ${ }^{1}$ Professor \& Head, ECE Department, Gudlavalleru Engineering College, Andhra Pradesh, India. \\ E-mail id:madduraju@yahoo.com \\ ${ }^{2}$ M.Tech (Embedded Systems), Gudlavalleru Engineering College,Andhra Pradesh, India. \\ E-mail id: gopidarsi@gmail.com
}

\begin{abstract}
The scope of networked embedded systems is rapidly increasing day by day due to their demand for monitoring and controlling appliances in home as well as industry. Embedded systems with networking provides web access for the industrial and research centers optimization. Data acquisition system with web access combindly gives the easy implementation of the system which uses ARM processor for control purpose and GPRS technology along with GSM is used for communication around the world. The real time operating system plays a crucial role in the system where the embedded device is booted with $\mu \mathrm{C} / \mathrm{OS}$, which is commonly used RTOS for hard real time systems. The proposed system consists of an $\mu \mathrm{COS}$ configured for real time scheduling in industrial applications which eliminates the need for server maintenance. This system enhances security by remotely monitoring various appliances.
\end{abstract}

\section{Indexing terms/Keywords}

ARM processor, Embedded Web Server, RTOS, GSM, Data Acquisition and Control System.

\section{Council for Innovative Research}

\author{
Peer Review Research Publishing System
}

Journal: INTERNATIONAL JOURNAL OF COMPUTERS \& TECHNOLOGY

Vol 10, No 4 


\section{INTRODUCTION}

The embedded technology is getting changing to the new design and new models and the computers are connecting to the World Wide Web through the internet. The data is getting exchanging among the computers which are longer distances through the internet connection to the World Wide Web. This feature facility provides us to transmit the data to the longer distance. For accessing the information from the remote location computers, need a web server. This will maintain the database of the information. People from the remote location using the IP address will access the information. The user can request a particular web page from the web server.

Computers with network facility can provide data transfer facility to the longer distances but as industries are need of sensing the industrial parameters and sending to the control room or to the remote locations computers are not suitable for the embedded applications. Also maintaining the computers for simple data transmissions will requires large space, more cost, and need of more memory for storing the data. As the current trend the use of embedded system devices is also increasing day by day in the different areas like handheld devices, home appliances control, and industrial equipment control and lot of these embedded system devices also supporting the networking features for low range and wide range data transmission. So using of the embedded system device with networking facility for remote data sensing and transmission will be an effective and good solution.

The Fig. 1 shows embedded web server architecture in the real industrial application and how to access it from the remote locations. So it introduces the concept of embedded web server. This architecture will overcome problems of data transmission and sensing parameter values in the industries by computers. In this architecture the embedded system device will have the networking connectivity with the help of Ethernet and TCP/IP protocol stack. Also industrial parameters can possible to read with the help of internal ADCs. And industrial equipments like motors can also controlled by $\mathrm{l} / \mathrm{O}$ port programming. Also these parameters can be viewed and controlled by opening the webpage at the Web server from the remote location using the IP address allocated to the embedded system device. Web server will be maintained in embedded system device and the information will be stored in the microcontrollers' internal memory. Microcontroller will run with the real time operating system.

The proposed system implemented here comes under embedded technology in association with network technology. The RTOS development is implemented in embedded C. When RTOS is incorporated into this system, more devices could be controlled and monitored and ensures portability and high reliability. In this current world scenario of globalization, where communication has become a necessity, this communication technology can be surely placed in the emerging trends of embedded systems technology[2].Through the Internet, could acquire the different real-time information, based on the information, could draft corresponding treatment options and implement remote Control through Internet. Comparing to the traditional personal computer-based Internet applications, the Embedded Internet application will surely lead us into a new realm.

\section{SYSTEM ARCHITECTURE}

\subsection{Embedded Web ServerArchitecture}

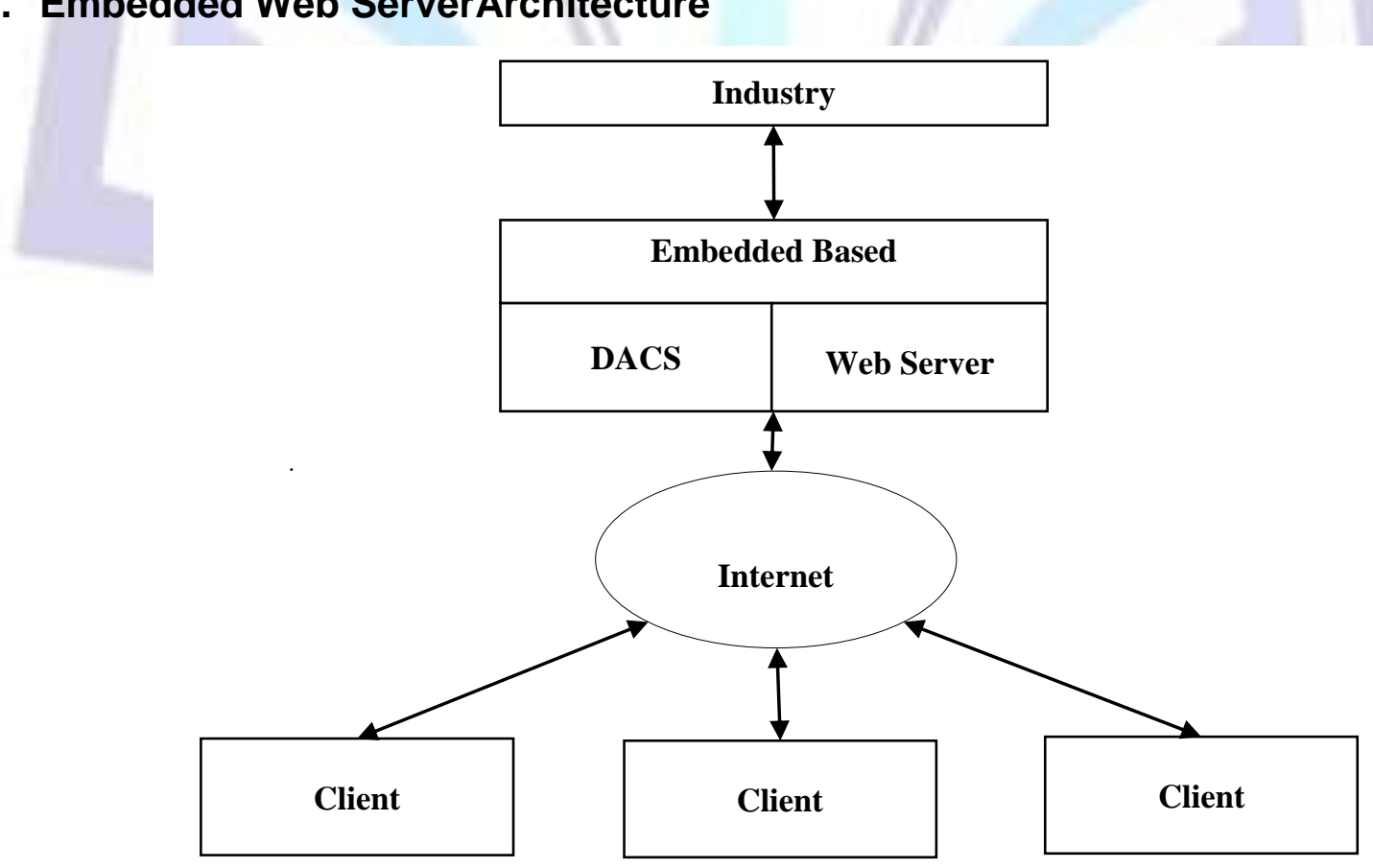

Figure 1: Embedded Web Server Architecture. 
Embedded web server architecture consists of an ARM processor that contains RTOS software for monitoring and controlling machines/systems. Embedded web servers are integral part of an embedded network. Fig. 1 shows the proposed concept of DACS with embedded web server presented on a single chip module. This is a single hardware it contains RTOS portable ARM processor [3]. Controlling the devices remotely and measuring signals is done by ARM processor. The system works in the DACS mode for measuring and embedded web server mode for sharing the data with the clients. The real time operating system manages all the tasks such as measuring signals, data base up-dation, sending HTML pages and connecting/communicating with new users etc.,

The RTOS manages all the required tasks in parallel and in small amounts of time [10]. The $\mu$ COS RTOS is configured for the priorities of the three sensors as shown in fig. 2. The scheduling for these sensors can be changed at the run time by configuring them to three different switches. Each sensor is provided with time slice. The three sensors complete their tasks within their time slices hence contributing to hard real time system. Web based management user interfaces using embedded web server have many advantages: ubiquity, user-friendly, low-development cost and high maintainability [4]. The different requirements of web server are high reliability, low resource usage, security, controllability and portability, which are suitable for most of the general web server technologies.

\subsection{Data Acquisition System}

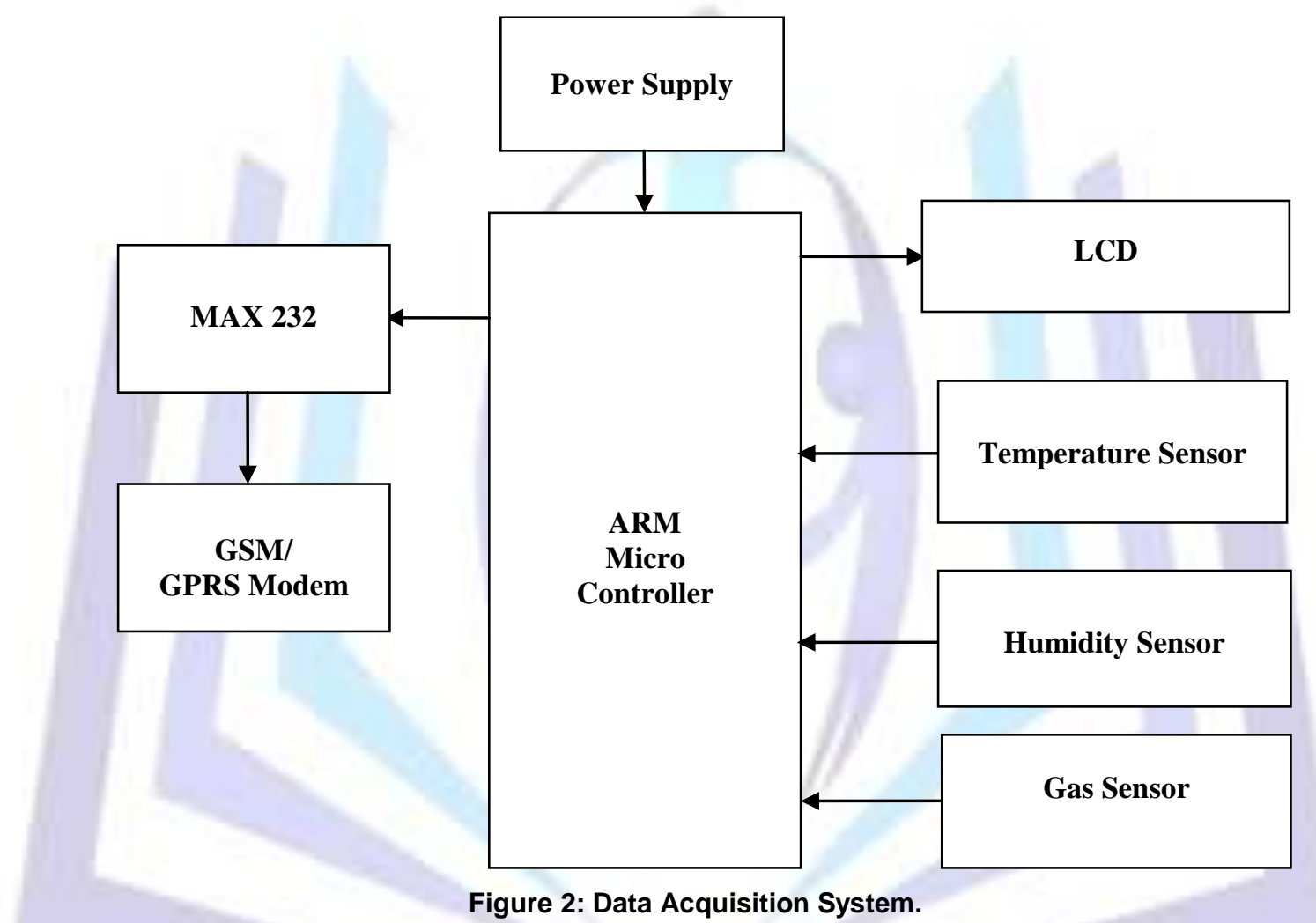

The data from the sensors is collected and maintained by the Data Acquisition and Control System by ARM processor [12]. In DACS mode the data is maintained in the data base. In Web Server mode, the data can be accessed by the client from anywhere across the world through internet [8]. The GSM modem is serially interfaced with the controller with the help of MAX 232 [5]. GSM modem shares data from the board to internet.

\section{IMPLEMENTATION}

The software coding for the hardware functionality is written in embedded $\mathrm{C}$ language in Keil software.

\subsection{Software}

The necessary requirement analysis is done on the RTOS, web server, TCP/IP and the target board. Based on the requirement analysis, the $\mu$ COS is configured, as per the target board. After configuring $\mu C O S$, generate the hex file with the necessary target options. The target is tested along with the RTOS, by booting it with the RTOS hex file. The application code for a specific RTOS is written and both the RTOS and web server are ported on the target. The web server application is tested for our desired function. The program flow and working of embedded web server is shown in fig. 3. The software consists of two major components. They are RTOS and HTML Pages.

\subsubsection{RTOS}


The application runs in the form of tasks. Tasks are created by each user connecting to the server. An RTOS is required to manage these tasks, which perform the operations in real time. The embedded web server is implemented using $\mu$ COS II a powerful but small RTOS kernel [11]. It is highly CPU independent and has been ported to numerous microprocessor platforms. Some of the simple codes and its explanation for $\mu \mathrm{COS}$ II are given below.

$>$ OS_TASK_CREATE_EN ( ): This function is used to have $\mu \mathrm{C} / \mathrm{OS}$-II manage the execution of tasks.

$>$ OS_TASK_CHANGE_PRIO_EN ( ): This function is used to change the priority of a task dynamically.

$>$ OSTimeDly (ticks): This function is used to delay execution of the currently running task until the specified number of system ticks expires.

$>$ OSSchedUnlock (void): This function is used to allow rescheduling of the tasks.

\subsubsection{HTML Pages}

HTML pages are used for data communication between the client and the server. In the embedded web server, web pages are selected as the media of interaction. HTML is used in designing these web pages. The html pages are saved on the board.

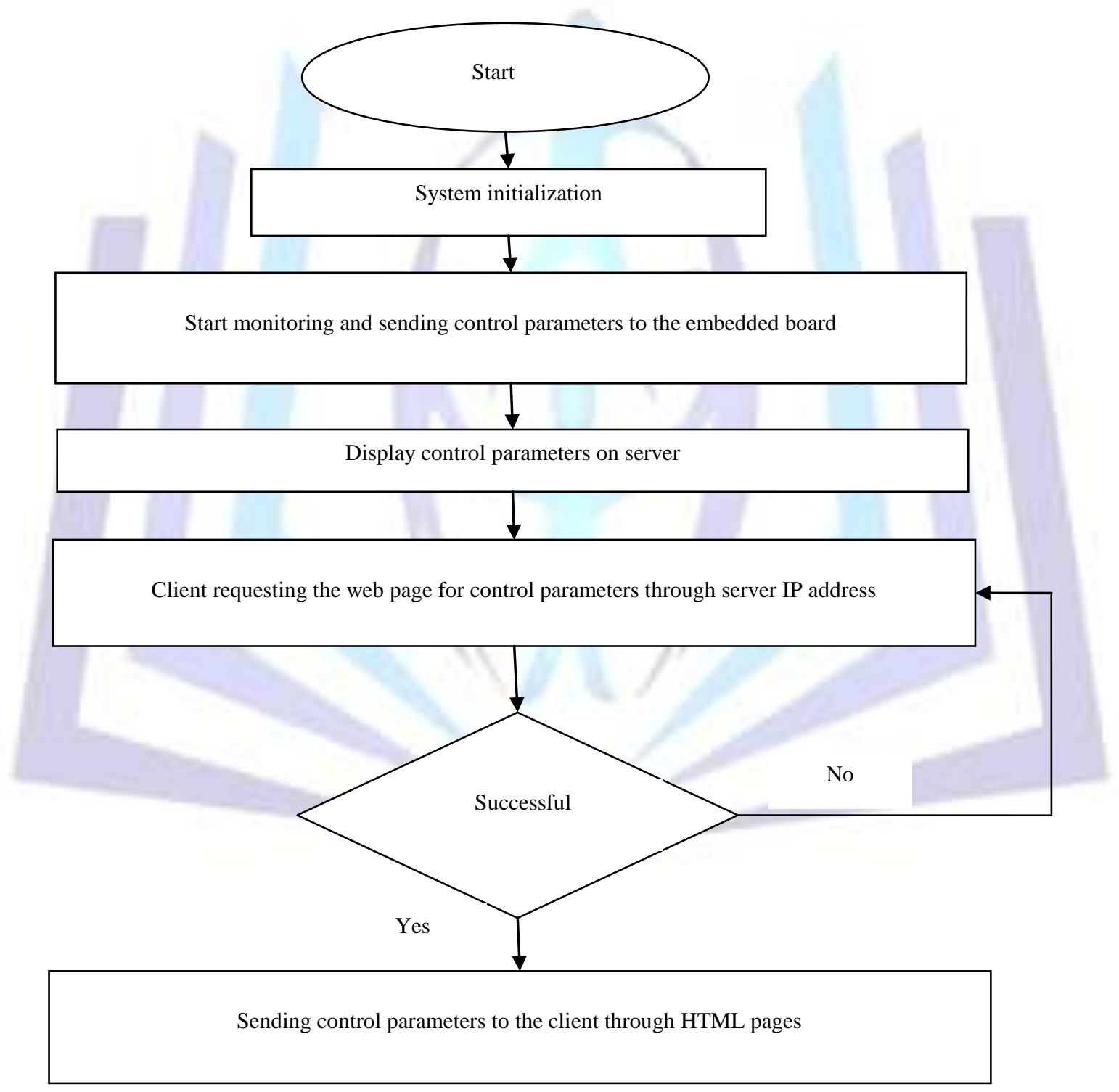

Figure 3: Flow chart for Embedded Web Server.

\subsection{Hardware}

The Fig. 3 gives the hardware components and their connections for the system. The hardware used for embedded web server is ARM based LPC2148 board [13]. The board itself consists of LCD for displaying sensor values. The three sensors i.e., temperature (LM35), gas and humidity sensors are interfaced to the embedded board. A GSM modem is 
connected to the board for establishing an internet connection [9]. Three switches are connected for configuring the RTOS scheduling i.e. for changing the priorities of the sensors dynamically at the run time.

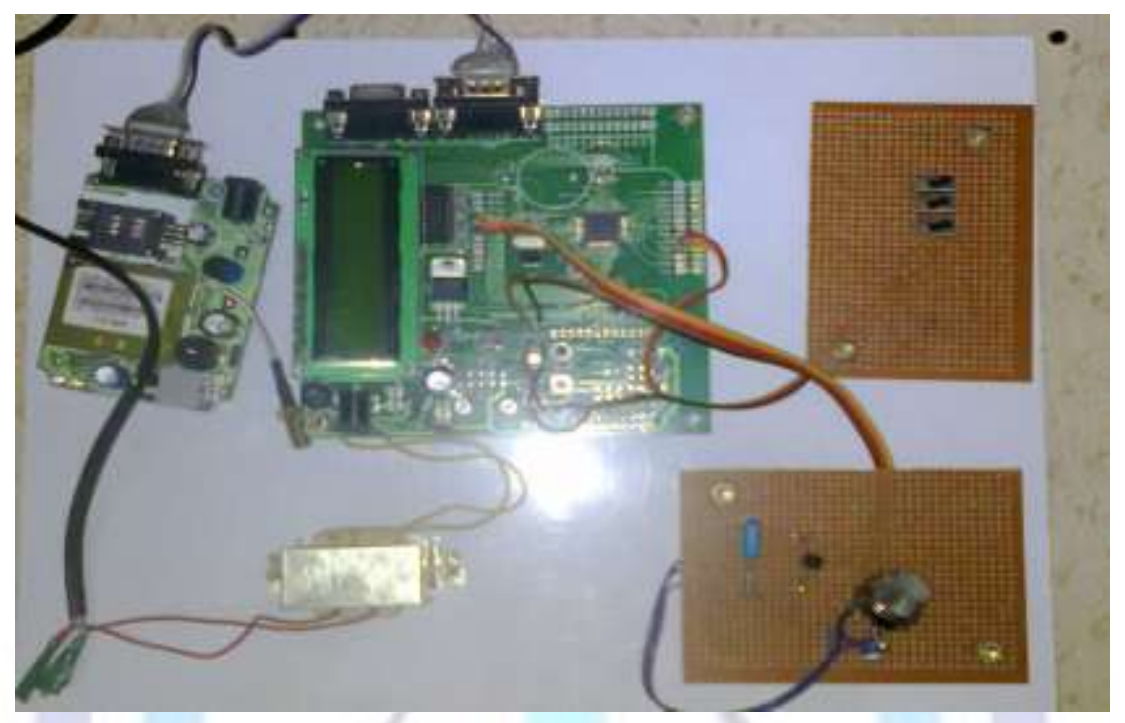

Figure 4: Photograph of the Embedded Web Server with Hardware components.

\section{TESTING OF EMBEDDED WEB SERVER}

Initially, the target is tested for the working of operating system. This is done by booting the target board using the hyper terminal. After the target is successfully booted with $\mu$ COS, it is tested over the network using ping command [6]. Now the embedded web server is responding to the clients. A request is made to the embedded web server, by giving the IP address of the server. The user has to enter valid IP to access the server. The operating system manages the request of the client and gives to the LAN controller of the client system. The LAN controller sends the request to the router which processes and checks for the system connected to the network with that particular IP address. If the IP address entered matches to that of the server, a request is sent to the LAN controller of the server to the client and hence a session is established between server and the client and the server starts sending the web pages to the client [7].

\section{RESULTS}

The execution results for the Embedded Web Server based DAC System are shown in fig. 5 and fig. 6.

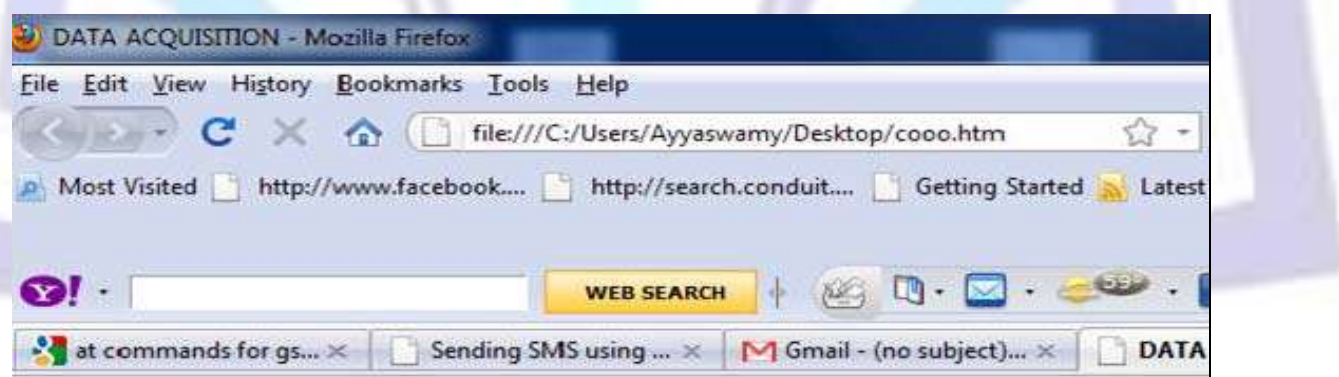

\section{Login id:}

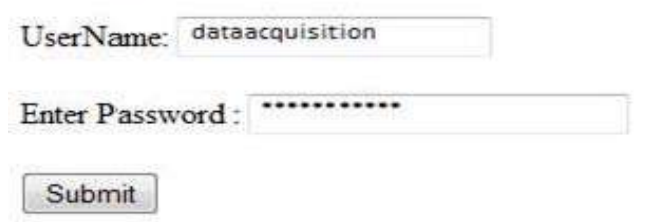

Figure 5: Online Processing Web Page.

Fig. 5 shows the online processing web page for the client authentication to access the embedded web server data. 


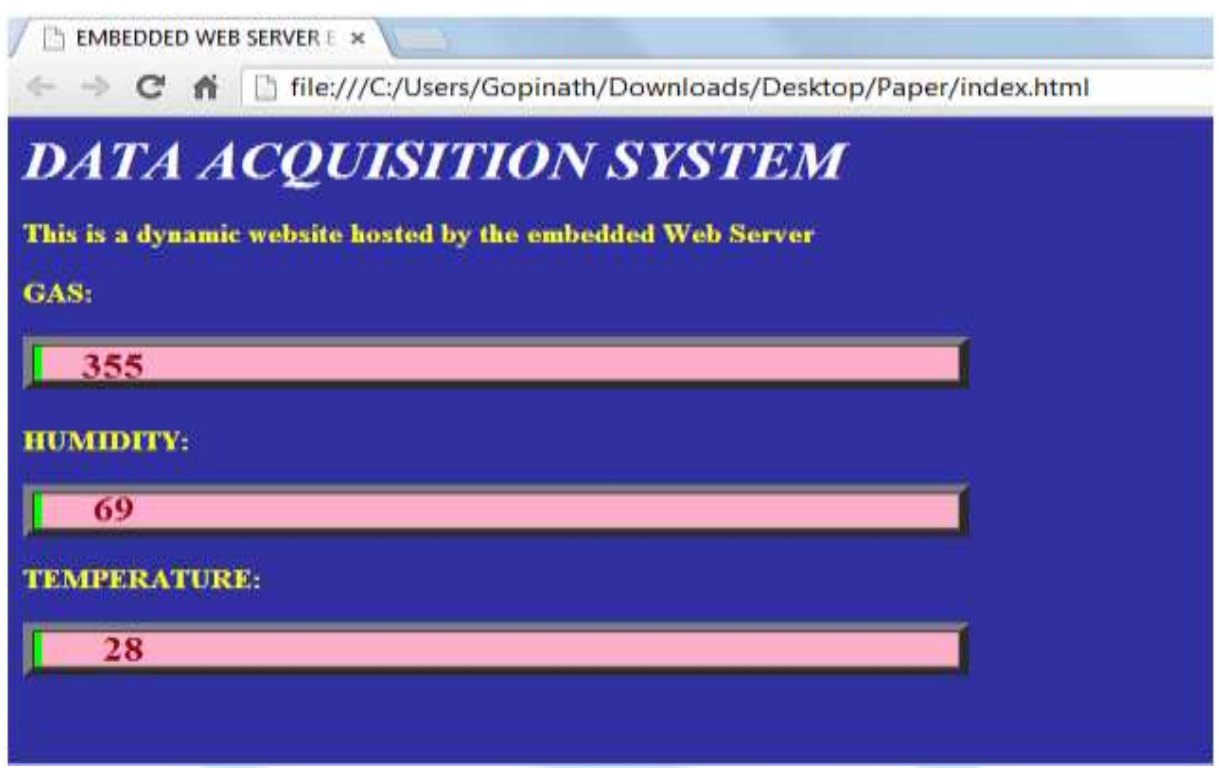

Figure 6: Clients requested Web Page.

The dynamic web page representing the sensor values in the Data Acquisition System maintained by the embedded web server is shown in fig. 6 . The client access the data dynamically through this web page send by the server. This is the simplest way of accessing data by the client. Moreover more than one client can access the data at the same time from different parts of the world.

\section{CONCLUSION}

The embedded web server based on data acquisition system has been designed which can be used for industrial applications and in many other places. Clients can monitor and control the parameters with an easy powerful user interface without additional hardware. The performance of the system can be increased by the architecture and Software contention. The web server can be connected to more serial devices at the same time with higher transmission rate with GSM technology. A low cost, simple data processing system is implemented which eliminates the need for server maintenance. A remote client only requires a common internet browser to carry out various parameters on real hardware.

\section{REFERENCES}

[1] S A.N.Sandeep, P.Malyadri -Embedded Web Server Based on DAC System Using ARM, in International Journal of Engineering Research and Applications,vol.2,no.4,July-August 2012, pp.

[2] AlenRajan, Aby K. Thomas -ARM Based Embedded Web Server for Industrial Applications. International Conference on Computing and Control Engineering (ICCCE 2012), 12 \& 13 April, 2012.

[3] Manivannan M, Kumaresan N. "Embedded web server \&gprs based advanced industrial automation using Linux rtos" Vol. 2(11), 2010, 6074-6081, ISSN: 0975-5462.

[4] Soumya Sunny P, Roopa .M. "Data Acquisition and Control System Using Embedded Web Server" International Journal of Engineering Trends and Technology- Volume3lssue3- 2012, pp.411-415.

[5] BaburaoKodavati, V.K.Raju, S.SrinivasaRao, A.V.Prabu, T.AppaRao, Dr.Y.V.Narayana "GSM and GPS based vehicle location and tracking system" International Journal of Engineering Research and Applications (IJERA) ISSN: 2248-9622 Vol. 1, Issue 3, pp.616-625

[6] V.BillyRakesh Roy, SanketDessai, and S. G.Shiva Prasad Yadav."Design and Development of ARM Processor Based Web Server" International Journal of Recent Trends in Engineering, Vol. 1, No. 4, May 2009, pp 94-98.

[7] K.Bharathreddy, Ch. RajendraPrasad, "The Embedded Web server based Electrical Ethernet Monitoring system using ARM", International Journal of Advanced Research in Computer and Communication Engineering Vol. 2, Issue 5, May 2013, pp 2292-2295.

[8] Klimchynski, —Extensible embedded Web server for internet-based data acquisition and control, in Proc. 3rd IEEE Int. Conf. Sensors, Vienna, Austria, Oct. 24-27, 2004, vol. 1, pp. 52-55.

[9] C. Bettstetter, H.-J. Vögel, and J. Eberspächer, -GSM phase 2+ Genera Packet Radio Service GPRS: Architecture, protocols, and air interface, IEEE Commun. Surveys Tuts, vol. 2, no. 4, pp. 2-14, Third Quarter 1999.

[10] M.Kamaraju, “A Novel design of Low cost Real Time Vehicle NavigationSystem,”International Journal of Engineering Science \&Technology(IJEST),Vol.2,No.8, August 2010, pp 3707-3711.

[11] RTOS Evaluation Project, —What makes a good RTOS, Dedicated Systems Experts, 2001. 
[Online].Available:http://www.dedicatedsystems.com

[12] RTOS website.http://www.ucos-ii.com/products/rtos/kernel/rtos.html

[13] ARM website. http://www.arm.com.

[14] Datasheet of LPC2148. http://www.nxp.com/documents/data sheet/LPC2141 424446 48.pdf

\section{Author' biography with Photo}

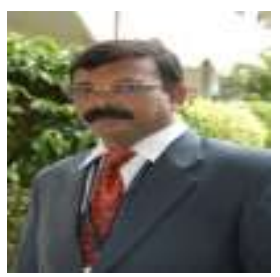

M.Kamaraju' ${ }^{1}$ obtained his Bachelor's Degree \& Master's degree from Andhra university and Ph.D from JNTUH, Hyderabad in the area of Low Power VLSI Design, Areas of interest are Microprocessors, Microcontrollers, Digital system Design, Embedded System Design, Low Power VLSI Design. He published 44 technical papers in national/ international journals/conferences. He reviewed number of papers for international journal and conferences. He is a Fellow of IETE and IE and member of IEEE. Presently working as Professor\& Head of ECE Department, Gudlavalleru Engineering College, Gudlavalleru, India and chairman of IETE, Vijayawada centre.

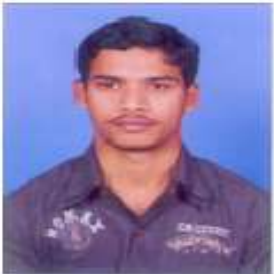

D.Gopinath ${ }^{2}$ obtained his Bachelor's Degree from QIS college of Engineering \& Technology, Ongole and completed PG Diploma in Embedded Systems Design from C-DAC, Chennai. His Areas of interest in Microprocessors, Microcontrollers, Embedded System Design,Low Power VLSI Design and VLSI CAD tools. He published one review paper on Embedded Webserver based on DAC System. Presently he is doing M.Tech embedded systems at Dept.of ECE, Gudlavalleru Engineering College,Gudlavalleru, India. 